\title{
GALAXY STRUCTURE AS A DRIVER OF THE STAR FORMATION SEQUENCE SLOPE AND SCATTER
}

\author{
Katherine E. Whitaker ${ }^{1,2,11}$, Marijn Franx ${ }^{3}$, Rachel Bezanson ${ }^{4,11}$, Gabriel B. Brammer ${ }^{5}$, Pieter G. van Dokkum ${ }^{6}$, \\ Mariska T. Kriek ${ }^{7}$, Ivo Labbé ${ }^{3}$, Joel Leja ${ }^{6}$, Ivelina G. Momcheva ${ }^{6}$, Erica J. Nelson ${ }^{6}$, Jane R. Rigby ${ }^{1}$, Hans-Walter Rix ${ }^{8}$, \\ Rosalind E. SKelton ${ }^{9}$, ArJen van der Wel ${ }^{8}$, ANd Stijn Wuyts ${ }^{10}$ \\ ${ }^{1}$ Astrophysics Science Division, NASA Goddard Space Flight Center, Code 665, Greenbelt, MD 20771, USA \\ 2 Department of Astronomy, University of Massachusetts-Amherst, Amherst, MA 01003, USA; kwhitaker@astro.umass.edu \\ ${ }^{3}$ Leiden Observatory, Leiden University, P.O. Box 9513, 2300 RA Leiden, The Netherlands \\ ${ }_{5}^{4}$ Steward Observatory, Department of Astronomy, University of Arizona, AZ 85721, USA \\ ${ }^{5}$ Space Telescope Science Institute, 3700 San Martin Drive, Baltimore, MD 21218, USA \\ ${ }^{6}$ Department of Astronomy, Yale University, New Haven, CT 06520, USA \\ ${ }^{7}$ Astronomy Department, University of California, Berkeley, CA 94720, USA \\ ${ }^{8}$ Max Planck Institut fur Astronomie, Königstuhl 17, D-69117 Heidelberg, Germany \\ ${ }^{9}$ South African Astronomical Observatory, P.O. Box 9, Observatory, Cape Town, 7935, South Africa \\ ${ }^{10}$ Max-Planck-Institut fr extraterrestrische Physik, Postfach 1312, Giessenbachstr, D-85741 Garching, Germany \\ Received 2015 June 25; accepted 2015 August 18; published 2015 September 22
}

\begin{abstract}
It is well established that (1) star-forming galaxies follow a relation between their star formation rate (SFR) and stellar mass $\left(M_{\star}\right)$, the "star formation sequence," and (2) the SFRs of galaxies correlate with their structure, where star-forming galaxies are less concentrated than quiescent galaxies at fixed mass. Here, we consider whether the scatter and slope of the star formation sequence is correlated with systematic variations in the Sérsic indices, $n$, of galaxies across the SFR $-M_{\star}$ plane. We use a mass-complete sample of 23,848 galaxies at $0.5<z<2.5$ selected from the 3D-HST photometric catalogs. Galaxy light profiles parameterized by $n$ are based on Hubble Space Telescope Cosmic Assembly Near-IR Deep Extragalactic Legacy Survey near-infrared imaging. We use a single SFR indicator empirically calibrated from stacks of Spitzer/MIPS $24 \mu \mathrm{m}$ imaging, adding the unobscured and obscured star formation. We find that the scatter of the star formation sequence is related in part to galaxy structure; the scatter due to variations in $n$ at fixed mass for star-forming galaxies ranges from $0.14 \pm 0.02$ dex at $z \sim 2$ to $0.30 \pm 0.04$ dex at $z<1$. While the slope of the $\log$ SFR $-\log M_{\star}$ relation is of order unity for disk-like galaxies, galaxies with $n>2$ (implying more dominant bulges) have significantly lower SFR $/ M_{\star}$ than the main ridgeline of the star formation sequence. These results suggest that bulges in massive $z \sim 2$ galaxies are actively building up, where the stars in the central concentration are relatively young. At $z<1$, the presence of older bulges within star-forming galaxies lowers global SFR $/ M_{\star}$, decreasing the slope and contributing significantly to the scatter of the star formation sequence.
\end{abstract}

Key words: galaxies: evolution - galaxies: formation - galaxies: high-redshift

\section{INTRODUCTION}

One of the outstanding problems in galaxy formation is understanding the causal relationship between the morphologies and star formation histories of galaxies. There is increasing evidence that galaxies with quiescent stellar populations have significantly smaller sizes and more concentrated light profiles than actively star-forming galaxies at fixed stellar mass out to $z=2.5$ (Kriek et al. 2009; Williams et al. 2010; Wuyts et al. 2011b; van der Wel et al. 2014). We know that quenching occurs, causing one population to migrate to the other. However, the process(es) that are primarily responsible for the shutdown of star formation are not well understood. It is yet unclear if the well-defined correlation between stellar mass and star formation rate (SFR; e.g., Brinchmann et al. 2004; Noeske et al. 2007; Whitaker et al. 2014; Schreiber et al. 2015, and numerous others) is driven by galaxy mass alone, or if some other parameter, such as surface density (Franx et al. 2008) or bulge mass (Lang et al. 2014), comes into play.

In the nearby universe, Abramson et al. (2014) find that increasing the galaxy bulge mass-fraction lowers the global specific SFR (sSFR $\equiv \mathrm{SFR} / M_{\star}$ ). Their working assumption is that star formation occurs in disks (Kennicutt 1998), and bulges

\footnotetext{
${ }^{11}$ Hubble Fellow.
}

are composed primarily of older stars. While the bulge contributes to the total stellar mass of the galaxy, it does not significantly contribute to the global star formation, thereby depressing the sSFR. This naturally predicts a flattening of the galaxy $\log$ SFR $-\log M_{\star}$ relation at the massive end where galaxies are bulge-dominated. Whitaker et al. (2014) measure a strong evolution in the slope of the star formation sequence at the massive end, consistent with a flat slope at $z<0.5$ and a slope close to unity at $z>2.5$. In other words, even at $z \sim 2$ we are seeing a depression in the global specific SFRs among massive galaxies.

Lang et al. (2014) decomposed the bulges and disks of massive high-redshift galaxies out to $z=2.5$. They speculate that significant bulge growth precedes the departure from the star formation sequence. Lang et al. further demonstrate that the bulge mass is a more reliable predictor of quiescence than the total stellar mass (see also Bell et al. 2012). To understand the regulation and quenching of star formation in galaxies across cosmic time, we must take these analyses one step further and connect the structural evolution of quiescent and star-forming galaxies with their sSFRs.

Before we can understand the details of how galaxies quench their star formation, we must first synthesize the properties of massive galaxies in a self-consistent manner. Such an analysis 
requires a robust quantification of the rest-frame optical galaxy structural properies and a single indicator for the total obscured and unobscured star formation in galaxies for the entire population. In this Letter, we combine Sérsic index measurements derived from Cosmic Assembly Near-IR Deep Extragalactic Legacy Survey (CANDELS)/WFC3 imaging with total UV + IR SFRs, using Spitzer/MIPS $24 \mu \mathrm{m}$ photometry, extending the $24 \mu \mathrm{m}$ stacking technique presented in Whitaker et al. (2014) to probe a range in structural parameters. We present the trends between galaxy $M_{\star}$, total sSFR, and $n$ at $0.5<z<2.5$, exploring the dependence of the slope and scatter of the star formation sequence on galaxy concentration.

In this Letter, we use a Chabrier (2003) initial mass function and assume a $\Lambda$ CDM cosmology with $\Omega_{\mathrm{M}}=0.3, \Omega_{\Lambda}=0.7$, and $H_{0}=70 \mathrm{~km} \mathrm{~s}^{-1} \mathrm{Mpc}^{-1}$. All magnitudes are given in the AB system.

\section{DATA AND SAMPLE SELECTION}

\subsection{Stellar Masses, Redshifts, and Rest-frame Colors}

We take advantage of the Hubble Space Telescope (HST)/ WFC3 and ACS photometric and spectroscopic datasets in five well-studied extragalactic fields through the CANDELS; (Grogin et al. 2011; Koekemoer et al. 2011) and the 3D-HST survey (Brammer et al. 2012). Using stellar masses, redshifts, and restframe colors from the 3D-HST $0.3-8 \mu \mathrm{m}$ photometric catalogs (see Skelton et al. 2014), we select samples of 9400, 8278, and 6170 galaxies greater than stellar masses of $\log M_{\star} / M_{\odot}=8.8$, 9.0, and 9.6 in three redshift intervals of $0.5<z<1.0$, $1.0<z<1.5$, and $1.5<z<2.5$. The galaxies are split into starforming and quiescent sub-samples based on their rest-frame $U-V$ and $V-J$ colors, following a modified definition of Whitaker et al. (2012a). ${ }^{12}$ We have used the Spitzer/IRAC color selections presented in Donley et al. (2012) to identify and remove luminous active galactic nuclei (AGNs); $3 \%$ of the sample were removed as AGN candidates. We note that the results of this Letter do not depend on this step. The final sample comprises 23,848 galaxies at $0.5<z<2.5$.

We indicate the stellar mass limits for which the majority of objects in the sample have $H_{F 160 W}<23.5$ (see Section 2.2). These limits are well above the mass-completeness limits presented in Tal et al. (2014), which are determined by comparing object detection in CANDELS/deep with a recombined subset of exposures reaching the CANDELS/wide depth. The stellar masses of star-forming galaxies have been corrected for contamination of the broadband fluxes from emission lines using the values presented in Appendix A of Whitaker et al. (2014). These corrections only become significant at $\log M_{\star} / M_{\odot}<9.5$ and $z>1.5$. For example, Whitaker et al. (2014) find that stellar masses of $\log M_{\star} / M_{\odot}=9.5$ are overestimated by $0.06(0.2)$ dex at $1.5<z<2.0(2.0<z<2.5)$ because of emission line fluxes.

Where available, we combine the spectral energy distributions with low-resolution HST/WFC3 G141 grism spectroscopy to derive grism redshifts with $\Delta z /(1+z)=0.3 \%$ accuracy (Brammer et al. 2012). We select the "best" redshift to be the spectroscopic redshift, grism redshift or the photometric redshift, in this ranked order depending on availability. Photometric redshifts comprise $62 \%$ (79\%) of the

\footnotetext{
${ }^{12}$ We no longer require $V-J<1.5$, similar to van der Wel et al. (2014), as this is a false upper limit imposed on the quiescent population. This effects $<1 \%$ of galaxies.
}

$0.5<z<1.5(1.5<z<2.5)$ sample, while $26 \%$ (19\%) have grism redshifts and $12 \%(2 \%)$ spectroscopic redshifts.

\subsection{Morphology}

The Sérsic (1968) index is a measure of the shape of the surface brightness profile of a galaxy, indicating the concentration of the light; it has been shown to correlate with quiescence (e.g., de Vaucouleurs 1948; Caon et al. 1993). At $1<z<3$, Bruce et al. (2014) demonstrate that $n=4$ corresponds to a bulge-to-disk ratio of about four in massive galaxies, where $\sim 80 \%$ of the light is in the bulge. Exponential disks have $n=1$ and bulge-to-disk ratios of about a quarter, with $20 \%$ of the light in the bulge. The transition from bulge-dominated to diskdominated occurs around $n=2$. We caution that we are not directly measuring the bulge mass-fractions, rather adopting $n$ as a proxy for bulge dominance. Furthermore, it is not clear whether this interpretation of $n$ holds at $z \gtrsim 1$.

$n$ is measured from $H S T / \mathrm{WFC} 3 J_{\mathrm{F} 125 \mathrm{~W}}$ at $0.5<z<1.5$ and $H_{\mathrm{F} 160 \mathrm{~W}}$ at $1.5<z<2.5$ (van der Wel et al. 2012). Simulations by van der Wel et al. (2012) show that the systematic errors in $n$ for all galaxy types are $<10 \%$ for $H_{F 160 W}<23.5$, thus motivating our mass limits. It is complicated to correct for the wavelength dependence of $n$ within the redshift bins, we do not correct for this effect but note that $n$ increases by $\sim 10 \%$ from 4500 to $8000 \AA$.

\subsection{Total SFRs}

Total star formation rates are derived from stacking analyses of Spitzer/MIPS $24 \mu \mathrm{m}$ photometry, following the procedure detailed in Whitaker et al. (2014). ${ }^{13}$ The Spitzer/MIPS $24 \mu \mathrm{m}$ images in the AEGIS field are provided by the Far-Infrared Deep Extragalactic Legacy (FIDEL) survey (Dickinson \& FIDEL Team 2007), COSMOS from the S-COSMOS survey (Sanders et al. 2007), GOODS-N and GOODS-S from Dickinson et al. (2003), and UDS from the Spitzer UKIDSS Ultra Deep Survey ${ }^{14}$ (SpUDS; PI: J. Dunlop). The analysis code uses a highresolution $J_{\mathrm{F} 125 \mathrm{~W}}+H_{\mathrm{F} 140 \mathrm{~W}}+H_{\mathrm{F} 160 \mathrm{~W}}$ detection image as a prior to model the contributions from neighboring blended sources in the lower resolution MIPS $24 \mu \mathrm{m}$ image (Labbé et al. 2010). All galaxies are "cleaned" of the contaminating flux of the neighboring sources before stacking. We refer the reader to Section 3 of Whitaker et al. (2014) for the full details of the MIPS $24 \mu \mathrm{m}$ stacking analyses. The SFRs derived for quiescent galaxies herein are upper limits, as the $24 \mu \mathrm{m}$ technique overestimates the SFRs for galaxies with $\log$ sSFR $<-10$ $\mathrm{yr}^{-1}$, but remains robust above this limit (Hayward et al. 2014; Utomo et al. 2014). For this reason, identifying star-forming galaxies from $24 \mu \mathrm{m}$-based sSFRs results in a significant fraction of interloping quiescent galaxies.

\section{RESULTS}

If increasing bulge mass-fractions result in a flattening of the star formation sequence at the massive end (Abramson et al. 2014), we should see a correlation between the $n, M_{\star}$, and sSFR. In Figure 1, we show the Sérsic indices of galaxies as a function of their stellar mass, color-coded by the UV+IR sSFRs for all galaxies (left), star-forming only (middle), and

\footnotetext{
13 Stacking is currently the only way to derive SFRs from a single indicator at low stellar masses or SFRs, separating this work from Wuyts et al. (2011b). 14 http://irsa.ipac.caltech.edu/data/SPITZER/SpUDS/
} 


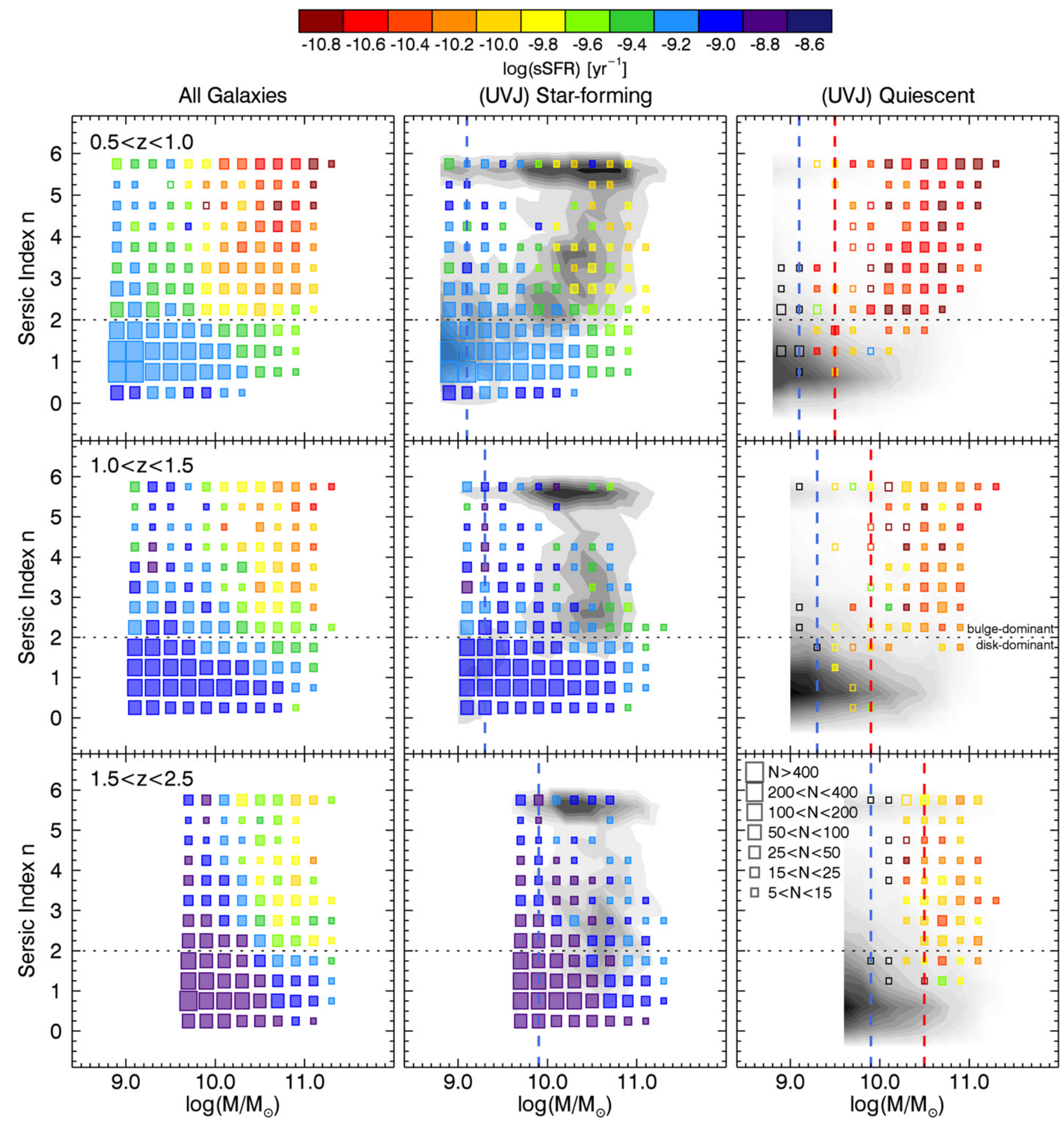

Figure 1. Sérsic indices of galaxies as a function of stellar mass, color-coded by the sSFRs derived from UV+IR stacking analyses in 0.2 dex bins of log $M_{\star} / M_{\odot}$ and 0.5 width bins of $n$. The vertical dashed lines correspond to the stellar mass limits down to which $n$ is robust for color-selected star-forming (blue) and quiescent (red) populations. At fixed stellar mass, galaxies with higher $n$ (implying prominent bulges) and $\log \left(M / M_{\odot}\right)>10$ have lower sSFRs, suggesting that the bulge formation is linked to quiescence. The size of the symbol depends on the number of galaxies, and the underlying grayscale demarcates the opposite population (e.g., star-forming galaxies in the quiescent panels).

quiescent only (right). Indeed, we find a decrease in the sSFRs for galaxies with high $n$ at $\log M_{\star} / M_{\odot}>10$. There does not appear to be any dependence of $n$ on the average sSFR for lowmass galaxies with $\log M_{\star} / M_{\odot}<10$.

Quiescent galaxies with $\log \left(M / M_{\odot}\right)>10$ tend to have $n>2$, consistent with bulge-dominated light profiles. Although this finding is not new, we do show for the first time that the analogous star-forming sub-population with similar $n$-values also have depressed sSFRs relative to the bulk of the starforming population. We also see a low-mass quiescent population at $0.5<z<1.0$ with similar (disk-like) Sérsic indices in Figure 1, albeit these galaxies lie close to or below the mass limit. This may suggest that lower-mass galaxies quench their star formation at later times through a more 

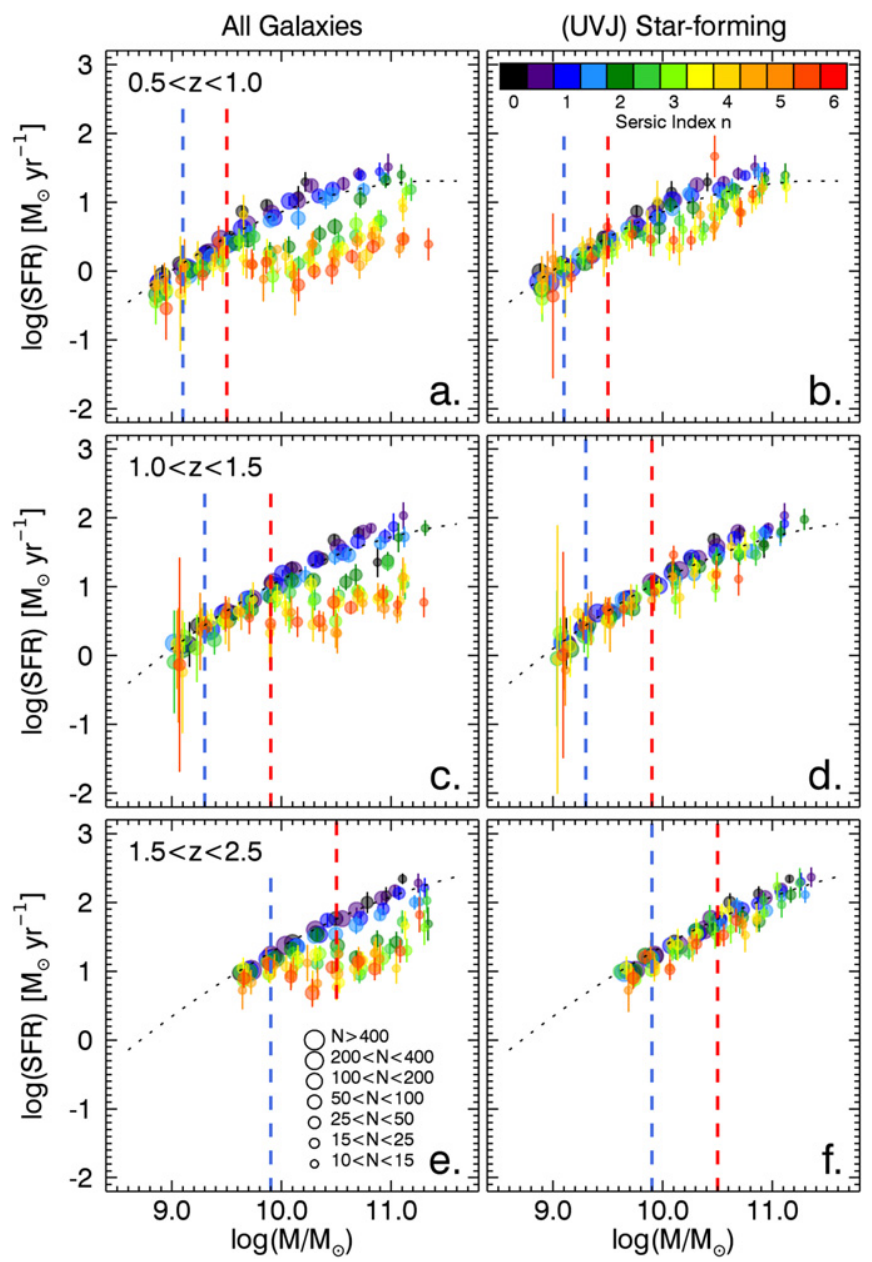

Figure 2. $\log (\mathrm{SFR})-\log M_{\star}$ relation of all galaxies (left) and color-selected star-forming (right) resulting from the stacking analysis across the $n-M_{\star}$ plane and vertical lines defined in Figure 1 . Each symbol is color-coded by $n$; the size of the symbol depends on the number of galaxies in the bin. The error bars are derived from a Monte Carlo bootstrap simulation of the stacking analyses. The average star formation sequence from Whitaker et al. (2014) is shown with dotted lines. Galaxies with significant bulges $(n>2)$ exhibit a shallower slope for the star formation-stellar mass relation.

gradual "slow-track" of gas depletion (see, e.g., Barro et al. 2013).

Figure 2 shows the $\log$ SFR- $\log M_{\star}$ projection of Figure 1, color-coded by $n$. At all redshifts, we see a clear correlation between SFR $/ M_{\star}$ and $n$ when considering both quiescent and star-forming galaxies (left panels). Although this result is primarily driven by the bulge-dominated, low sSFR quiescent population, we do see a weaker trend for star-forming galaxies only. The more concentrated the light profile of a galaxy (high$n$ ), the more likely that galaxy is to have a depressed sSFR relative to the star formation sequence.

Assuming $n$ is a proxy for bulge-to-disk ratio, we see evidence to support the idea of Abramson et al. (2014): the slope of the star formation sequence is correlated with the build-up of bulges. We note, however, that even galaxies with $n=1$ exhibit some curvature of the star formation sequence, suggesting an additional mechanism acting to suppress star formation in massive galaxies.

Figure 3 shows the slope of $\log \mathrm{SFR}-\log M_{\star}$ relation measured above the star-forming mass limits. The error bars include the uncertainty in the slope measurement itself and $n$, where the latter is calculated from 100 bootstrap simulations perturbing $n$ by the random errors and repeating the analysis. A summary of the best-fit parameters is found in Table 1 . We find similar general trends when selecting star-forming galaxies by $\log (\mathrm{sSFR})>-10 \mathrm{yr}^{-1}$. Whitaker et al. (2012b) demonstrated that a steeper slope of the star formation sequence is measured when selecting blue star-forming galaxies (e.g., Peng et al. 2010). Here, we show explicitly that by selecting galaxies with prominent disks (which often have blue rest-frame optical colors), we measure a slope close to unity across the full range in stellar mass and redshift. We confirm similar results by Wuyts et al. (2011b), and emphasize the critical importance of understanding selection biases.

\section{DISCUSSION}

The aim of this Letter is to connect rest-frame optical measurements of the $n-M_{\star}$ relation with total sSFRs for a single SFR indicator from a purely empirical standpoint. We can thereby connect galaxy structure and star formation to understand the observed bimodal distribution of galaxies across cosmic time. We measure a systematic decrease in the global sSFRs of galaxies with increasing $n$. This flattening of the star formation sequence directly reflects the bimodality of the two stellar populations: quiescent galaxies tend to be bulgedominated with significantly lower sSFRs, whereas starforming galaxies are typically disk-dominated with sSFRs consistent with the main ridge of the star formation sequence (confirming Wuyts et al. 2011b). The measured SFR- $M_{\star}$ relation for galaxies with $n=2$ tracks the best-fit relations of Whitaker et al. (2014) in Figure 2. Galaxies with $n<2$ have a slightly steeper (but still curved) relation, whereas galaxies with $n>2$ show a significantly flattened relation. We also see that $n$ correlates with the color bimodality of the populations (Figure 4, Panel A).

When considering star-forming galaxies only, pure exponential-disk galaxies exhibit a higher slope of the $\log$ SFR $-\log M_{\star}$ relation than those with a significant bulge component. This result is somewhat dependent on how starforming is defined: UVJ-selected star-forming galaxies at $z<1$ with $n>2$ have rest-frame colors close to the quiescent region (Panel B, Figure 4). If we shift the quiescent box redward in $V-J$ by $+0.1-0.3 \mathrm{mag}$ and repeat the analysis, we find the same correlation between the slope of the star formation sequence and $n$ at $z<1$. This is because high- $n$ galaxies drop out of the sample altogether but variations in the slope from $n=0.5$ to $n=3$ remain. The galaxies that lie in this intermediate region of rest-frame color space may be an extension of the quiescent population on the verge of shutting down star formation (e.g., Belli et al. 2015, Papovich et al. 2015; M. Yano et al. 2015, in preparation). They have more concentrated light profiles and lower sSFRs than typical starforming galaxies.

For star-forming galaxies at $z>1$, the slope of the star formation sequence instead depends weakly on $n$ (right panel, Figure 3 ). The correlation is not statistically significant above $n>1$. Shifting the UVJ selection redward increasingly weakens the trend. Perhaps the larger photometric errors of quiescent galaxies at high- $z$ scatter some into the intermediate parameter space, which get removed by this test. The $n>2$ star-forming galaxies well separated from the UVJ line show a similar range of colors to $n<2$ star-forming galaxies (compare 


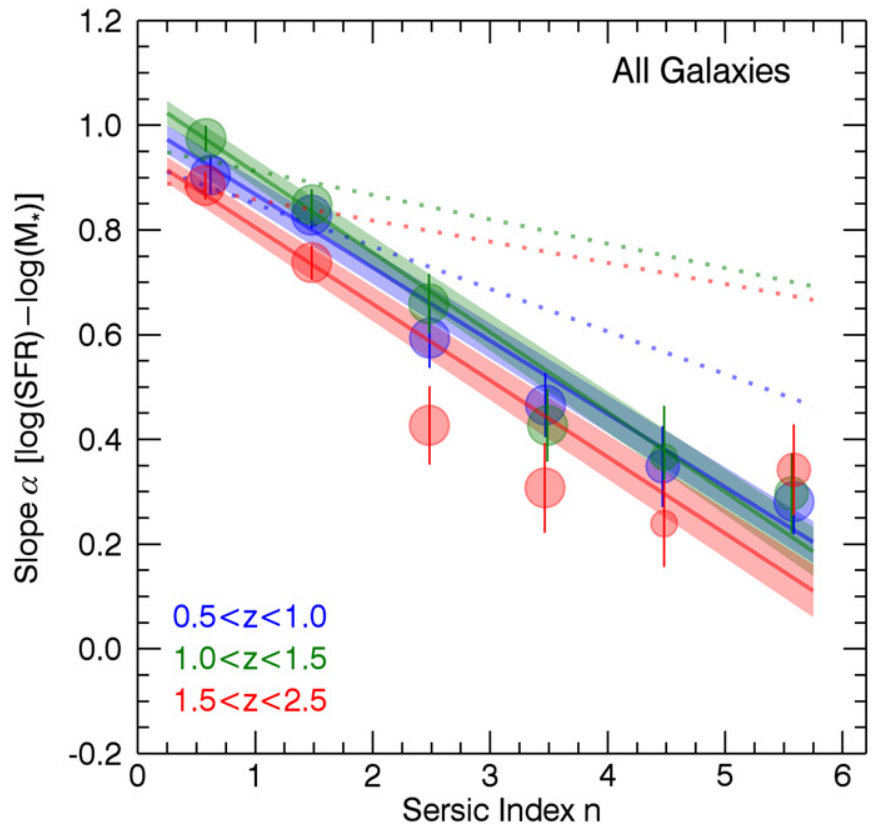

(a) All Galaxies

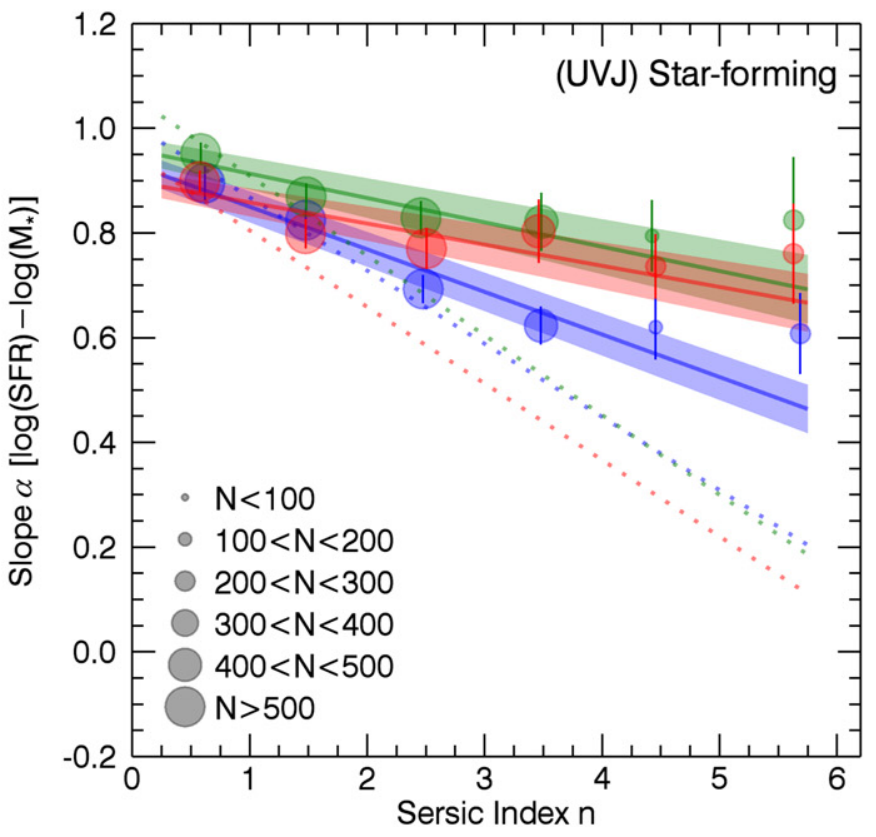

(b) (UVJ) Star-forming Galaxies

Figure 3. Best-fit linear slope $\alpha$ of the $\log$ SFR- $\log M_{\star}$ relation above the star-forming mass limits depends strongly on $n$ when both star-forming and quiescent galaxies are considered. This trend is weaker for star-forming galaxies only, and may signify active bulge-formation among the most massive galaxies at $z \sim 2$, where the stars in the central concentration are younger. The symbol size represents the number of galaxies $N$. Dotted lines are the best-fit relation from the opposite panel for reference.

Table 1

Linear Fits to the Slope $\alpha$ of the $\log$ SFR- $\log M_{\star}$ Relation vs. Sérsic index

\begin{tabular}{lccccc}
\hline \hline & \multicolumn{2}{c}{ All Galaxies } & & \multicolumn{2}{c}{ (UVJ) Star-forming } \\
\cline { 2 - 3 } \cline { 5 - 6 } Redshift Range & $a$ & $b$ & & $a$ & $b$ \\
\hline $0.5<z<1.0$ & 1.01 & $-0.14 \pm 0.01$ & & 0.93 & $-0.08 \pm 0.01$ \\
& \pm 0.03 & & \pm 0.03 & \\
$1.0<z<1.5$ & 1.06 & $-0.15 \pm 0.01$ & & 0.96 & $-0.05 \pm 0.02$ \\
& \pm 0.03 & & & \pm 0.03 & \\
$2.5<z<2.5$ & 0.95 & $-0.15 \pm 0.01$ & & 0.90 & $-0.04 \pm 0.01$ \\
& \pm 0.03 & & & \pm 0.03 & \\
& & & &
\end{tabular}

Note. Linear fit coefficients parameterizing the evolution of the slope, $\alpha$, of the $\log$ SFR- $\log M_{\star}$ relation as a function of Sérsic index, $n$, where $\alpha=a+b n$.

Panels D and G, Figure 4). They may also have similar sSFRs, although $n>3$ star-forming galaxies are not prevalent at the highest masses. Disk-dominated star-forming galaxies are neither preferentially dusty or dust-free; their range in colors in Figure 4 likely reflects the inclination angle through which we view the disk (Patel et al. 2012). The process of forming a significantly massive bulge at $z>1$ appears to be connected to a transformation in the rest-frame optical galaxy structure and color before the decrease of the global sSFR of galaxies. Panel $\mathrm{D}$ in Figure 4 demonstrates that the galaxy morphology is in place at $z \sim 2$ before the reddening of the rest-frame colors associated with passive evolution.

The observation that the correlation between the slope of the star formation sequence and $n$ is weaker among star-forming galaxies at $z>1$ may tell us something about bulge formation. Traditional bulges may already be in place at $z<1$. For example, van Dokkum et al. (2014) find little growth in the centers of massive galaxies below $z<0.8$, but at higher redshifts there is growth at all radii. Furthermore, Nelson et al.
(2012) demonstrate that (new) star formation at $z \sim 1$ occurs at all radii in exponential disks. In other words, bulges at $z \lesssim 1$ may already be composed of older stars.

The picture is less clear at higher redshifts; it may be that massive galaxies are actively building up their bulges. From Figures $1-3$, we see that bulge-dominated galaxies at high- $z$ exist, but do not seem to have a significantly lower global sSFR. If the bulges of massive $z \sim 2$ galaxies are actively forming, the stars will still be young and there should not yet be a strong depression in global sSFRs. Choi et al. (2014) derived stellar ages for a large sample of quiescent galaxies at $0.1<z<0.7$ that are consistent with an equivalent single-burst star formation epoch of $z \sim 1.5$, supporting the idea that $z \sim 2$ is an important epoch for the bulge formation for massive galaxies. This does not however tell us anything about how bulges grow. We cannot tell if clumps of stars form insitu and migrate to the bulge due to disk instabilities (e.g., Noguchi 1999; Elmegreen et al. 2008), if they are the result of a rich merger history (e.g., De Lucia et al. 2011), or some other mechanism.

The best way to decompose the star formation histories of $z \sim 2$ galaxies will be with future spatially resolved studies. For example, Nelson et al. (2015) find that the radial $\mathrm{H} \alpha$ profiles of galaxies above the ridge of the star formation sequence are enhanced at all radii, whereas galaxies below have suppressed star formation at all radii. Their results suggests that a global process regulates gas accretion, and the scatter in the star formation sequence is real. It is however unclear why the amount of gas accretion depends on disk mass instead of total mass. In this work, we demonstrate that this scatter may be related in part to $n$. In Figure 2, the scatter of the SFRs between $10<\log \left(M / M_{\odot}\right)<11$ for all galaxies ranges from $0.34 \pm 0.09 \mathrm{dex}$ at $1.5<z<2.5$, to $0.43 \pm$ $0.05 \mathrm{dex}$ at $1.0<z<1.5$, and $0.48 \pm 0.02 \mathrm{dex}$ at $0.5<z<1.0$. When considering UVJ-selected star-forming 

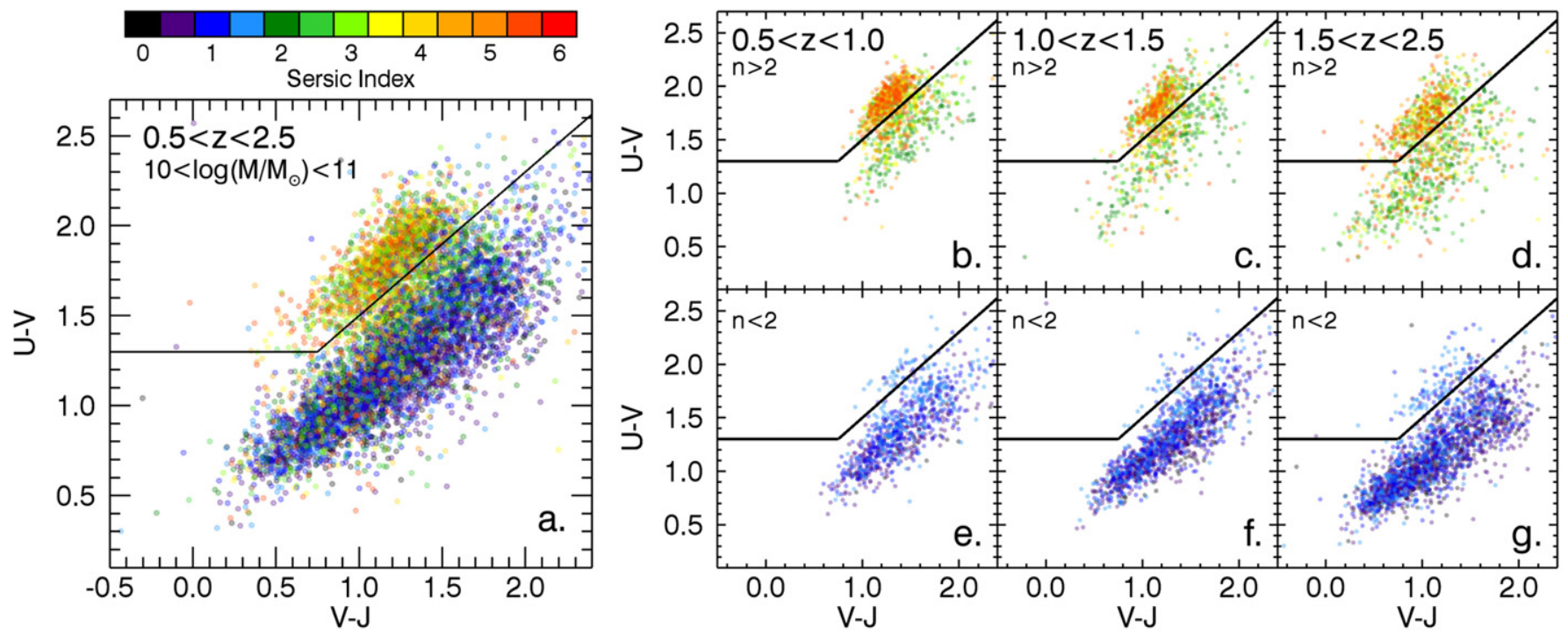

Figure 4. Rest-frame $U-V$ and $V-J$ colors also reflect a bimodality in the Sérsic index (panel (a)), with bulge-dominated galaxies predominatly inhabiting the quiescent region and disk-dominated galaxies in the star-forming region. There do not exist strong trends within the quiescent and star-forming populations. The notable exception is the cross-over region between these two populations, where galaxies tend to be bulge-dominated, matching the quiescent population, while still residing in the star-forming color-color parameter space at $z<1$ (panel (b)). Conversely, at $z>1.5$, star-forming galaxies with high Sérsic indices cover the full colorcolor parameter space (panel (d)).

galaxies only, these values are $0.14 \pm 0.02 \mathrm{dex}$ at $1.5<z<2.5,0.21 \pm 0.05$ dex at $1.0<z<1.5$, and 0.30 $\pm 0.04 \mathrm{dex}$ at $0.5<z<1.0$. Compared to the roughly constant 0.34 dex observed scatter of the star formation sequence (Whitaker et al. 2012b), we show that this scatter is driven in part by real differences in $n$. Random errors will introduce $\sim 0.05$ dex scatter, and evolution of the average SFR within the redshift bins $\sim 0.11-0.14$ dex (Whitaker et al. 2012b). It may be that a significant portion of the remaining scatter is correlated with variations in $n$ among galaxies. However, we are unable to decompose the relative contributions of intrinsic and systematic scatter at present, and note that the $24 \mu \mathrm{m}-L_{\mathrm{IR}}$ conversion itself could introduce as much 0.25 dex of scatter (Wuyts et al. 2011a).

These results support the idea that we are witnessing the rapid build-up of bulges at $z \sim 2$ in massive galaxies. A prominent bulge may be an important condition for quenching to occur (e.g., Bell et al. 2012), at least in massive galaxies. Franx et al. (2008) demonstrated that surface density and inferred velocity dispersion are better correlated with SSFR and color than stellar mass. We will explore these dependencies in an upcoming paper (K. Whitaker et al. 2015, in preparation).

We thank the anonymous referee for a highly constructive report. This work is based on observations taken by the 3DHST Treasury Program (GO 12177 and 12328) with the NASA/ESA $H S T$, which is operated by the Associations of Universities for Resarch in Astronomy, Inc., under NASA contract NAS5-26555. This research was supported in part by an appointment to the NASA Postdoctoral Program at the Goddard Space Flight Center, administered by Oak Ridge Associated Universities through a contract with NASA and by NASA through Hubble Fellowship grant HST-HF2-51368 awarded by the Space Telescope Science Institute, which is operated by the Association of Universities for Research in Astronomy, Inc., for NASA, under contract NAS5-26555.

\section{REFERENCES}

Abramson, L. E., Kelson, D. D., Dressler, A., et al. 2014, ApJL, 785, L36 Barro, G., Faber, S. M., Pérez-González, P. G., et al. 2013, ApJ, 765, 104 Bell, E. F., van der Wel, A., Papovich, C., et al. 2012, ApJ, 753, 167 Belli, S., Newman, A. B., \& Ellis, R. S. 2015, ApJ, 799, 206 Brammer, G. B., van Dokkum, P. G., Franx, M., et al. 2012, ApJS, 200, 13 Brinchmann, J., Charlot, S., White, S. D. M., et al. 2004, MNRAS, 351,1151

Bruce, V. A., Dunlop, J. S., McLure, R. J., et al. 2014, MNRAS, 444, 1660 Caon, N., Capaccioli, M., \& D’Onofrio, M. 1993, MNRAS, 265, 1013 Chabrier, G. 2003, PASP, 115, 763

Choi, J., Conroy, C., Moustakas, J., et al. 2014, ApJ, 792, 95

De Lucia, G., Fontanot, F., Wilman, D., et al. 2011, MNRAS, 414, 1439

de Vaucouleurs, G. 1948, AnAp, 11, 247

Dickinson, M., \& FIDEL Team 2007, BAAS, 39, 822

Dickinson, M., Papovich, C., Ferguson, H. C., et al. 2003, ApJ, 587, 25

Donley, J. L., Koekemoer, A. M., Brusa, M., et al. 2012, ApJ, 748, 142

Elmegreen, B. G., Bournaud, F., \& Elmegreen, D. M. 2008, ApJ, 688, 67

Franx, M., van Dokkum, P. G., Schreiber, N. M. F., et al. 2008, ApJ, 688,770

Grogin, N. A., Kocevski, D. D., Faber, S. M., et al. 2011, ApJS, 197, 35

Hayward, C. C., Lanz, L., Ashby, M. L. N., et al. 2014, MNRAS, 445,1598

Kennicutt, R. C., Jr. 1998, ARA\&A, 36, 189

Koekemoer, A. M., Faber, S. M., Ferguson, H. C., et al. 2011, ApJS, 197, 36 Kriek, M., van Dokkum, P. G., Franx, M., et al. 2009, ApJL, 705, L71

Labbé, I., González, V., Bouwens, R. J., et al. 2010, ApJL, 708, L26

Lang, P., Wuyts, S., Somerville, R. S., et al. 2014, ApJ, 788, 11

Nelson, E. J., van Dokkum, P. G., Brammer, G., et al. 2012, ApJL, 747, L28

Nelson, E. J., van Dokkum, P. G., Förster Schreiber, N. M., et al. 2015, ApJ, submitted (arXiv:1507.03999)

Noeske, K. G., Weiner, B. J., Faber, S. M., et al. 2007, ApJL, 660, L43

Noguchi, M. 1999, ApJ, 514, 77

Papovich, C., Labbé, I., Quadri, R., et al. 2015, ApJ, 803, 26

Patel, S. G., Holden, B. P., Kelson, D. D., et al. 2012, ApJL, 748, L27

Peng, Y.-j., Lilly, S. J., Kovač, K., et al. 2010, ApJ, 721, 193

Sanders, D. B., Salvato, M., Aussel, H., et al. 2007, ApJS, 172, 86

Schreiber, C., Pannella, M., Elbaz, D., et al. 2015, A\&A, 575, A74

Sérsic, J. L. 1968, Atlas de Galaxias Australes (Córdoba, Argentina: Observatorio Astronómico)

Skelton, R. E., Whitaker, K. E., Momcheva, I. G., et al. 2014, ApJS, 214, 24

Tal, T., Dekel, A., Oesch, P., et al. 2014, ApJ, 789, 164

Utomo, D., Kriek, M., Labbé, I., et al. 2014, ApJL, 783, L30 
van der Wel, A., Bell, E. F., Häussler, B., et al. 2012, ApJS, 203, 24 van der Wel, A., Franx, M., van Dokkum, P. G., et al. 2014, ApJ, 788, 28 van Dokkum, P. G., Bezanson, R., van der Wel, A., et al. 2014, ApJ, 791, 45 Whitaker, K. E., Franx, M., Leja, J., et al. 2014, ApJ, 795, 104

Whitaker, K. E., Kriek, M., van Dokkum, P. G., et al. 2012a, ApJ, 745, 179
Whitaker, K. E., van Dokkum, P. G., Brammer, G., et al. 2012b, ApJL, 754, L29

Williams, R. J., Quadri, R. F., Franx, M., et al. 2010, ApJ, 713, 738

Wuyts, S., Förster Schreiber, N. M., Lutz, D., et al. 2011a, ApJ, 738, 106

Wuyts, S., Förster Schreiber, N. M., van der Wel, A., et al. 2011b, ApJ, 742, 96 